Friedhelm HoFfmanN ${ }^{1}$

\title{
Die Aufgabe 10 des Moskauer mathematischen Papyrus
}

Im Moskauer mathematischen Papyrus ${ }^{2}$ hat die Aufgabe 10 auf den Kolumnen 18-20 seit Struves Erstedition schon immer die besondere Aufmerksamkeit der Forscher erregt ${ }^{3}$. Denn Struve verstand den Text so, daß hier die Oberfläche einer Halbkugel berechnet wird ${ }^{4}$. Eine solche Leistung hätte niemand der ägyptischen Mathematik zugetraut. Zunächst war man verblüfft und staunte ${ }^{5}$. Ein zaghafter Einwand kam allerdings von Archibald: Warum wird $\pi$ auf andere Weise berechnet als im P. Rhind ${ }^{6}$ ? Peet hingegen wollte den Text emendieren und den Ausfall einer Zahlenangabe annehmen. Nach seinem Verständnis wird entweder die gekrümmte Halbzylinderaußenfläche oder die Halbkreisfläche berechnet ${ }^{7}$. Peets Auffassung schloß sich Wolf $a^{8}{ }^{8}$. Er vermochte aber auch nicht zu entscheiden, ob ein Halbkreis oder ein Halbzylinder berechnet wird. Eine weitere Deutung lieferte Neugebauer, der die Aufgabe so verstand, daß überhaupt nur die näherungsweise Berechnung der Oberfläche eines kuppelförmigen Speichers Gegenstand der Aufgabe 10 ist'. In der Folgezeit herrschte Ratlosigkeit, welche Auffassung zutrifft, doch hielt man allgemein die Berechnung der Halbkugeloberfläche für eher unwahrscheinlich ${ }^{10}$. In jüngerer Zeit hat sich Gillings wieder für die ursprüngliche Ansicht stark gemacht und sieht wie Struve die Aufgabe 10 des Moskauer Papyrus als Beleg dafür an,

${ }^{1}$ Ich danke J. Gesellensetter, L. Goldbrunner, S. Hallmann, H. Hussy und D. Ostermeyer, die im Wintersemester 1993/94 an meiner Übung über mathematische Texte teilgenommen haben und mit denen ich das hier behandelte Problem schon diskutieren konnte, für ihre Gesprächsbereitschaft und Anregungen. H. Beinlich und meiner Frau Beate danke ich sehr herzlich für die kritische Durchsicht des Manuskripts.

${ }^{2}$ Ed. Struve, W. W.: Mathematischer Papyrus des Staatlichen Museums der Schönen Künste in Moskau. Berlin 1930 (= Quellen und Studien zur Geschichte der Mathematik. Abteilung A: Quellen Bd. 1).

3 Transkription und Übersetzung siche am Ende dieses Aufsatzes.

${ }^{4}$ Struve op. cit. S. $157 \mathrm{ff}$.

${ }^{5}$ Struve op. cit. S. 169; Heath, T. L. In: Nature 127 (1931) S. 584; Neugebauer, O. In: Göttingische gelehrte Anzeigen 193 (1931) S. 30; Vogel, K. In: Archiv für Geschichte der Mathematik, der Naturwissenschaften und der Technik 13 (1931) S. 457.

${ }^{6}$ Archibald, R. C. In: Isis 16 (1931) S. 154. In unserem Text wird $\pi / 2 \cdot$ Durchmesser (D) angenähert als $2 \mathrm{D}-1 / 9 \cdot 2 \mathrm{D}=16 / 9 \cdot \mathrm{D}$ (vgl. den Text im Anhang). Der P. Rhind berechnet in Aufgabe 50 dagegen die Kreisfläche als $(8 / 9 \cdot \mathrm{D})^{2}$.

${ }^{7}$ Peet, T. E.: A Problem in Egyptian Geometry. In: JEA 17 (1931) S. $100 \mathrm{ff.}$

${ }^{8}$ Wolf, W. In: AfO 7 (1931-32) S. 205

9 Neugebauer, O.: Die Geometrie der ägyptischen mathematischen Texte. In: Quellen und Studien zur Geschichte der Mathematik[,] Astronomie und Physik. Abteilung B: Studien 1,4. Berlin 1931, S. 427 f.; Neu ge ba u er, O.: Vorlesungen über Geschichte der antiken mathematischen Wissenschaften. Bd. 1: Vorgriechische Mathematik. 2. Aufl. Berlin/Heidelberg/New York 1969 (= Die Grundlehren der mathematischen Wissenschaften in Einzeldarstellungen mit besonderer Berücksichtigung der Anwendungsgebiete 43), S. $136 \mathrm{f}$.

${ }^{10}$ Bessel-Hagen, E. In: OLZ 35 (1932) Sp. 398; van der Waerden, B. L.: Erwachende Wissenschaft. Ägyptische, babylonische und griechische Mathematik. 2. Aufl. Basel/Stuttgart 1966 (= Wissenschaft und Kultur 8), S. 53 f. Reineke, W.-F.: Die mathematischen Texte der Alten Ägypter. Teil II Berlin 1964 (Diss.), S. 156 ff. neigt gleichfalls Peets Ansicht von der nb.t als Halbzylinder zu, erwähnt aber nicht, daß Peet als Alternative auch den Halbkreis als Deutung der nb.t zur Diskussion stellt. 
daß die Ägypter sehr wohl die Oberfläche einer Halbkugel berechnen konnten ${ }^{11}$. Das Lexikon der Ägyptologie Bd. 3 Sp. 1242 folgt Gillings und begnügt sich in Anmerkung 34 auf Sp. 1245 mit einem unentschiedenen „Berechnung des Halbzylinders bzw. der Halbkugel“ und verweist auf Gillings ${ }^{12}$.

Ich selbst finde, daß in der bisherigen Diskussion keineswegs alle Argumente, die eine Klärung herbeiführen können, genannt worden sind. Da die Aufgabe 10 des Moskauer mathematischen Papyrus für die Geschichte der Mathematik so überaus bedeutend ist, wenn die Ägypter wirklich fast anderthalb Jahrtausende vor den Griechen die Halbkügeloberfläche korrekt berechnet haben, verdient das Problem eine erneute Behandlung.

Folgende Deutungen also sind bisher gegeben worden:

- Berechnung einer Halbkugeloberfläche

- Berechnung einer Halbzylindermantelfläche

- Berechnung einer Halbkreisfläche

- angenäherte Berechnung der Oberfläche einer langgestreckten Kuppel.

Der Vollständigkeit halber sollte man auch die Möglichkeit überprüfen, daß eine Kreissegmentfläche berechnet wird $^{13}$, ist doch der etymologische Nachfolger des Wortes nb.t, das das berechnete Objekt in der Aufgabe 10 bezeichnet, in den demotischen mathematischen Texten als nby „Kreissegment“ belegt ${ }^{14}$. Noch andere Möglichkeiten, eine 0 18.5) bzw. $\square \bigcirc \circlearrowright$ (so 18.2) zu deuten, sehe ich nicht.

Das Wort nb.t/nby macht nun offenbar eine Bedeutungsveränderung durch, die es unmöglich macht, vorderhand $\mathrm{zu}$ entscheiden, welches geometrische Gebilde im Moskauer Papyrus gemeint ist: In den Pyramidentexten $\int 557$ ist die $n b . t$ aus Pflanzen hergestellt und steht parallel $\mathrm{zu}$ einer Tasche $(k, 3 r)$, meint also auf jeden Fall einen dreidimensionalen Gegenstand. Das gleiche gilt natürlich für die „Hügel o. ä.“, die das Wort $n b . t$ bezeichnen kann ${ }^{15}$.

In den demotischen mathematischen Texten bezeichnet das Wort hingegen das Kreissegment, also ein zweidimensionales Objekt. Vermutlich ist für die Übertragung des Wortes die Art der Darstellung des $n b$-Zeichens in der Hieroglyphenschrift verantwortlich zu machen: Sie zeigt den Korb von der Seite, was ihn wie ein Kreissegment aussehen läßt. Die ursprüngliche Bedeutung „Korb“ scheint andererseits im demotischen Teil des Rosetta-Dekrets Z. 27 bewußt zu sein, da das Wort dort mit Hausdeterminativ geschrieben ist ${ }^{16}$. Zudem zeigt Dendera Mar. I 31, eine Parallele zum gerade erwähnten Pyramidenspruch, daß man auch sonst noch in spätzeitlichen Texten - abgesehen von nb.t in der Verbindung ho ว.m-nb.wt - auf nb.t als räumliches Gebilde stoßen kann.

Für die Aufgabe 10 des Moskauer Papyrus bleibt damit zunächst weiterhin alles offen. Wir kommen aber einen Schritt weiter, wenn wir kunstgeschichtliche Gegebenheiten mit einbeziehen. Es gibt nämlich dreidimensionale Darstellungen des $n b$-Zeichens. So kann es, wie die oben herangezogene Stelle des Rosetta-Dekrets beschreibt, unter eine Kobra gesetzt werden (im

\footnotetext{
${ }^{11}$ Gillings, R. J.: The Area of the Curved Surface of a Hemisphere in Ancient Egypt. In: Australian Journal of Science 30.4 (1967) S. $113 \mathrm{ff}$; ders.: Mathematics in the Time of the Pharaohs. New York 1982, S. $194 \mathrm{ff}$.

${ }^{12}$ Dort muß es aber „Australian“ statt „Austrian" heißen.

${ }^{13}$ Von Peet op cit. S. 103 Fn. 1 kurz gestreift.

${ }^{14}$ Parker, R. A.: Demotic Mathematical Papyri. London 1972 (= Brown Egyptological Studies 7), Aufg. 36 Zeilen 11 und 13 und Aufg. 37 Zeilen 4 und 6; dazu ibid. S. 45 Kommentar zu Zeile 11.

${ }^{15}$ Vercoutter, J.: Les Haou-Nebout ( 8 ). In: BIFAO 46 (1947) S. 156 ff. H. Hussy hat mich freundlicherweise an diesen Aufsatz erinnert.

${ }^{16}$ Allerdings werden auch Wörter wie 't "Gegend“ mit Hausdeterminativ geschrieben, obwohl sie doch eher etwas Zweidimensionales bezeichnen.
} 
nb.ty-Namen etwa, aber auch sonst). Diese Zeichenkombination kommt nun auch als Amulett vor und ist dann dreidimensional gestaltet. Die $n b . t$ wird dabei als Halbzylinder geformt ${ }^{17}$.

Ferner dient die $n b$-Hieroglyphe in Verbindung mit dem Haus zur Schreibung des Namens der Göttin Nephthys. Wird diese, wie es oft geschieht, vollplastisch dargestellt, wird so, wie etwa bei Isis der Thron auf dem Kopf als richtiger dreidimensionaler Thronsitz gebildet wird, auch die $n b$-Hieroglyphe vollplastisch wiedergegeben. Diese Darstellungen zeigen das Zeichen klar als Halbzylinder, nicht als Halbkugel ${ }^{18}$.

Denkbar ist immerhin die Möglichkeit, daß die erwähnten plastischen Darstellungen der nb-Hieroglyphe unrichtige Umsetzungen eines Halbkreises sind, der seinerseits nichts anderes als die ägyptische Darstellungsweise einer Halbkugel im Flachbild ist: Um einen Halbkreis vollplastisch wiedergeben zu können, mußte man ihm eine gewisse Dicke geben, um ihn überhaupt dreidimensional darstellen zu können.

Gegen diesen Einwand, der gewissermaßen um zwei Ecken herum argumentiert, spricht meiner Meinung nach dreierlei. Erstens ist es nicht einsichtig, warum man das $n b$-Zeichen dann so dick machen sollte. Sonnenscheiben beispielsweise bleiben in vollplastischer Darstellung ja auch relativ dünn und werden keineswegs zu flachen Zylindern. Zweitens gibt es bei nb.t „Bergrücken“ auch das Determinativ $\hookrightarrow^{19}$. Es zeigt klar, daß die nb.t ein langgestrecktes Gebilde sein muß, mithin keine Halbkugel sein kann. Ob schließlich bei einer nb.t des Himmels $^{20}$ auch an die halbzylindrisch gewölbten Decken mit den Darstellungen der Himmelsgöttin in Königsgräbern im Tal der Könige ${ }^{21}$ erinnert werden darf? Drittens hat das $n b$-Zeichen nicht einfach eine glatte Oberfläche, die es haben müßte, wenn es bloß die verdickte Wiedergabe von etwas Zweidimensionalem wäre. Vielmehr wird, wie die oben erwähnten Skulpturen erkennen lassen, in der Regel eine Querstreifung oder ein kariertes Muster angebracht, was fraglos als Hinweis auf die Tatsache, daß die nb.t geflochten ist, zu verstehen ist. Wenn sie aber geflochten ist, ist sie dreidimensional. Dann besteht aber kein Grund zu zweifeln, daß die Halbzylinderform die eigentliche Gestalt der nb.t ist.

Nach all dem dürfte die Auffassung der nb.t im P. Moskau als Halbkugel oder als kuppelförmiges Gebilde (auch ein rotationssymmetrischer Körper) ausscheiden. Es ist aber

17 Reisner, M. G. A.: Amulets. Bd. 2 Kairo 1958 (= Cat. Gén.), Taf. XIII CG 13158. Kleinere Figürchen werden leicht unscharf (ibid. Taf. XIV CG 13225).

${ }^{18} \mathrm{Zu}$ Darstellungen vgl. Carter, H.: Tomb of Tut-Ankh-Amen Discovered by the Late Earl of Carnarvon and Howard Carter. Bd. 3 New York 1963, Taf. VII; Daress y, G.: Statues de divinités. Bd. 2 Kairo 1905 (= Cat. Gén.), Taf. XLVII CG 38926, 38930, 38931, 38937b; ibid. Taf. LVIII CG 39221; ibid. Taf. LIX CG 39260 und 39262; Eggebrecht, A. (Hg.): Suche nach Unsterblichkeit. Totenkult und Jenseitsglaube im Alten Ägypten. Hildesheim und Mainz 1990, S. 85; Reisner op cit. Taf. XXVII CG 12974; Seipel, W.: Ägypten. Götter, Gräber und die Kunst. 4000 Jahre Jenseitsglaube. Bd. 1 Linz 1989 (= Kataloge des OÖ. Landesmuseums. Neue Folge Nr. 22; Bd. 1), Nr. 123; Grewenig, M. M./Seipel, W.: Götter Menschen Pharaonen. 3500 Jahre ägyptische Kultur. Meisterwerke aus der Ägyptisch-Orientalischen Sammlung des Kunsthistorischen Museums Wien. Speyer 1993, Nr. 183 und 223; Wildung, D./Schoske, S.: Nofret - Die Schöne. Die Frau im Alten Ägypten. Mainz 1984, S. 193 (= Daress y op. cit. Taf. LVIII CG 39220); u. v. a. m. Unscharf und leicht abgerundet sind Daressy op. cit. Taf. XLVII CG 38927 und Taf. LIX CG 39254; Reisner op. cit. Taf. XXXI CG 13505 und 13517 ; Petrie, W. M. F.: Amulets. Warminster 1972 (= Nachdruck der Ausgabe 1914) Taf. XXVII Nr. 152b, 154b und $154 \mathrm{c}$; und andere. Eine grob halbkugelige Ausbildung findet sich nur ganz ausnahmsweise bei späten und sehr kleinen Fayence-Figürchen (etwa Petrie loc. cit. Nr. 155b und Doetsch-Amberger, E.: Ägyptische Sammlung. Köln 1987, Nr. 105) und wird als Nachlässigkeit des Handwerkers zu werten sein.

${ }^{19}$ Vercoutter op. cit. S. $141 . \quad{ }^{20}$ Vercoutter op. cit. S. 135, 141 und 148.

${ }^{21}$ Die Deckform ist sehr deutlich beispielsweise bei Hornung, E.: Tal der Könige. Die Ruhestätte der Pharaonen. Darmstadt 1983, S. 134 Abb. 107 und 108 (Grab Ramses' VI.) zu sehen. 
noch keineswegs erwiesen, daß die nb.t auch im Kontext des mathematischen Moskauer Papyrus dreidimensional ist.

Allerdings wird nach dem bisher Gesagten Struves Deutung des Wortes $\stackrel{c}{d}$ in 18.3 als „Erhaltung“ > ,größter Kreisumfang“" unwahrscheinlich, da es einen „größten Kreis“ an einer nb.t, die, wie wir gesehen haben, ein Halbkreis (oder Kreissegment) oder ein Halbzylinder sein muß, gar nicht geben kann. Tatsächlich hat schon Peet op. cit. S. 101 das Wort als neben $t_{p}-r_{3}$ genannte zweite Dimension der nb.t aufgefaßt. Dem würde ich mich anschließen wollen. Zunächst scheint mir nämlich die Transkription bei Struve nicht korrekt. Das Determinativ

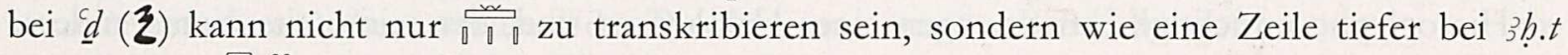
genausogut $\frac{\pi}{0} \mathbb{0}^{22}$.

Ist das richtig, sollten wir ein Wort ' $\underline{d}$ finden können, das eine Fläche oder Erstreckung o. ä. bezeichnet. Das ist tatsächlich der Fall: $\underline{d}\left(\underline{m}, \quad\right.$ u. ä.) meint das Land am Wüstenrand ${ }^{23}$, und sein demotischer Nachfolger $c^{c}$ bedeutet „Ufer, Festland, Seite, Gegend ${ }^{\text {“24 }}$. Dieses Wort ist geeignet, neben $t p-r_{3}$, „Mündung “ eine zweite Dimension der $n b . t$ anzugeben. Da $t p-r_{3}$ wie bei Dreiecken gebraucht werden dürfte, kommen folgende beiden Möglichkeiten für $\underline{d}$ in Frage:
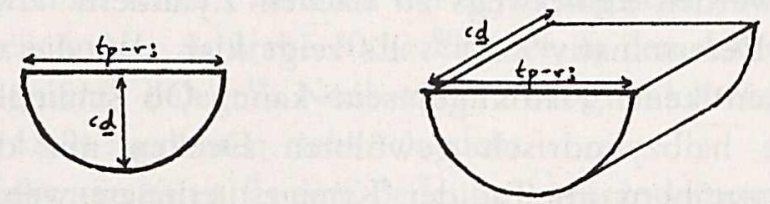

Geht man bei ${ }^{c} \underline{d}$ von der Bedeutung „Land am Wüstenrand, Seite“ aus, kann $\underline{d}$ eigentlich nur die Höhe des Halbzylinders meinen, der ja in der Tat eine gewisse Ähnlichkeit zum Niltal mit links und rechts aufsteigenden Wüstenrändern hat. Überdies wäre bei der Berechnung der Halbkreisfläche die Angabe einer weiteren Dimension neben dem Durchmesser unnötig. Denn man wird davon ausgehen dürfen, daß die alten Ägypter, wenn sie die Kreisfläche aus dem Durchmesser berechnen konnten, auch die halbe Kreisfläche allein aus dem Durchmesser ermitteln konnten. Nun bietet der Text aber mit der durch Parallelismus gekennzeichneten Formulierung

nib.t

$$
\begin{aligned}
& m \text { tp-r }{ }^{2} \\
& \text { r } \\
& 4 \frac{1}{2} m \stackrel{c}{d}
\end{aligned}
$$

allen Grund zu der Annahme, daß $m$ tp-r's und $m{ }^{c} \underline{d}$ zwei durch $r$,zu, auf ${ }^{6 * 6}$ verbundene verschiedene Dimensionsangaben sind ${ }^{25}$. Man muß dann vor $m$ tp-r, eine vom Schreiber vergessene Zahl ergänzen. Da wir für die nb.t rotationssymmetrische Körper ausschließen konnten, somit nur Halbzylinder und Halbkreis oder Kreissegment geblieben sind, muß die ausgelassene Zahl den Wert 41/2 (für Halbzylinder) oder 9 (für Halbkreis und Kreissegment) gehabt haben, um auf die Flächenzahl von 32 zu kommen.

Wir haben andererseits gerade gesehen, daß die nb.t des P. Moskau kaum ein Halbkreis sein kann, da dann überhaupt die Angabe einer zweiten Dimension unmotiviert erscheint. Es bliebe aber die Möglichkeit, daß hier der Spezialfall eines solchen Kreissegmentes berechnet wird, bei dem die Höhe gleich der halben Schnittkantenlänge ist. Dieser spezielle Fall wäre natürlich

\footnotetext{
${ }^{22}$ Struve op. cit. S. 162 Fn. 2; Peet op. cit. S. 104 Fn. 3 und Taf. XIII.

${ }^{23}$ Wb I S. 239,6. $\quad{ }^{24}$ Erichsen, W.: Demotisches Glossar. Kopenhagen 1954, S. 74.

${ }_{25}$ So schon Peet op. cit.; zu dieser Funktion von $r$ vgl. auch P. Rhind Nr. 45 Z. 2, wo drei Dimensionen mit $r$ zueinander in Beziehung gesetzt werden.
} 
der Halbkreis ${ }^{26}$, aber es mag durchaus denkbar sein, daß er einmal als Spezialfall eines Kreissegments aufgefaßt und entsprechend berechnet wird. Die Zahl 9, mit der in Zeile 5 von Kolumne 18 die Ausrechnung beginnt, wäre dann genau die in Zeile 2 derselben Kolumne zu ergänzende Zahlenangabe der Dimension $t_{p-r}$. A Auch $g s$ in Zeile 6 spricht nicht unbedingt dagegen. gs muß ja nicht nur „Hälfte“ bedeuten, sondern kann auch „Seite“ heißen ${ }^{27}$, womit sich durchaus ein Kreissegment bezeichnen ließe.

Zwei Punkte aber sprechen gegen die Deutung der nb.t als Kreissegment. Einmal ist nicht einsichtig, wie das Wort $\stackrel{c}{d}$, Rand" dazu verwendet werden kann, die Höhe eines Kreissegmentes zu bezeichnen. Dies dürfte tatsächlich mit der sonst für ${ }^{c} d$ bekannten Bedeutung unvereinbar

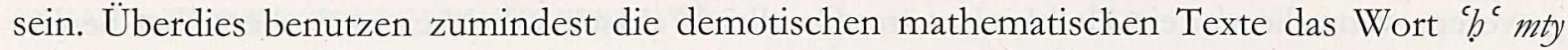
„Mittelhöhe“ zur Bezeichnung der Höhe eines Kreissegments ${ }^{28}$. Das zweite Gegenargument betrifft die Art und Weise, wie die Rechnung vor sich geht. Es ist völlig klar, daß in ihr eine ägyptische Näherung für $\pi$ vorkommt. In den demotischen mathematischen Texten ${ }^{29}$ hingegen wird die Fläche eines Kreissegments als

(Höhe+Breite) : $2 \cdot$ Höhe

angenähert. $\pi$ kommt nicht vor. Ob die Ägypter auch in früherer Zeit nach dieser Formel gerechnet haben, wissen wir natürlich nicht; auch nicht, ob sie vorher überhaupt Kreissegmente berechnet, haben.

Und doch können wir aus der Art der Berechnung, denke ich, entnehmen, daß die Ägypter gewissermaßen mit zwei verschiedenen $\pi$ gearbeitet haben. Das eine kommt z. B. im P. Rhind 48 und 50 bei der Ausrechnung der Kreisfläche als (8/9 Durchmesser) $)^{2}$ vor. Hier findet ein multiplikatorisches Verfahren statt, was natürlich auf eine Fläche, eben die Halbkreisfläche, führt. In unserem Text dagegen wird von einer Zahl ihr Neuntel subtrahiert $(18.5 \mathrm{ff} .)^{30}$. Ein solcher Rechenschritt führt in der Anschauung auf ein lineares Maß, nicht auf eine Fläche. Ich bin daher eher geneigt anzunehmen, daß die zwei unterschiedlichen Verfahren zur Annäherung von $\pi$ durch die unterschiedlichen berechneten Gebilde bedingt.sind, als daß der P. Rhind grundsätzlich ein anders Verfahren benutzt als der P. Moskau.

Mit anderen Worten: Wenn im P. Moskau mit Hilfe von $\pi$ aus dem Durchmesser eine lineare Größe ermittelt wird, kann das nur der Kreisumfang (bzw. der halbe Kreisumfang) sein ${ }^{31}$. Wird aber der Kreisumfang berechnet, kann die nb.t nur ein Halbzylinder sein, dessen gekrümmte Mantelfläche berechnet wird; dazu braucht man ja diese Größe. Bei der Ausrechnung der Kreisfläche fängt man mit dem Kreisumfang nichts an.

Schließlich geben die Zeilen 5 und 6 der Kolumne 18 einen weiteren Fingerzeig. Dort wird offenbar die Begründung dafür gegeben, warum die Rechnung mit der Zahl 9 ihren Ausgangspunkt nimmt. Die Erklärung wäre wohl überflüssig, wenn die Zahl 9 eine in der Aufgabenstellung schon genannte Größe gewesen wäre. (Das wäre bei einer Halbkreisberechnung der Fall gewesen.) Offenbar ist die Erklärung aber nötig, und der Grund dürfte sein, daß hier schon ein erster Rechenschritt stillschweigend vollzogen ist. Das würde die Alternative (Halbzylinder) stützen: Wenn in der Aufgabe 10 des Moskauer Papyrus die gekrümmte

\footnotetext{
${ }^{26}$ Wie schon Peet op. cit. S. 103 Fn. 1 richtig bemerkt, kann, wenn die nb.t eine Fläche ist, wegen des Rechenergebnisses (32) kein anderes Kreissegment als der Halbkreis vorliegen.

${ }^{27} \mathrm{~Wb}$ V S. $191 \mathrm{ff}$.

28 Parker op. cit. Nr. 37,6.

${ }^{29}$ Parker op. cit. S. 44.

30 Darüber hat sich schon Archibald loc. cit. gewundert.

${ }^{31}$ Wieso die Berechnung des Kreisumfangs schwerer sein soll als die der Kreisfläche, wie Gillings, R. J.: Mathematics in the Time of the Pharaohs, S. $197 \mathrm{f}$. impliziert, leuchtet mir nicht ein.
} 
Halbzylinderaußenfläche berechnet wird, müssen, wie wir gesehen haben, beide Dimensionsangaben in der Aufgabenstellung 41/2-gewesen sein. Wenn der Schreiber gleich mit 9 rechnet, einer Zahl, die nicht in der Aufgabenstellung vorkam, muß er dies erklären.

Tatsächlich leitet im Moskauer mathematischen Papyrus ḅr-ntt auch sonst immer eine nachträgliche Begründung für einen unmittelbar zuvor erfolgten Rechenschritt ein. So wird in $15.5 \mathrm{ff}$. und 26.1 begründet, warum man mit der Zahl 2 multiplizieren muß. In 38.3 und 38.4f. wird eine Konvertierung von Scheffelbruchteilen in Bruchzahlen erklärt. Aufgrund dieser Strukturierung der Aufgaben ist es ziemlich klar, daß die Zahl 9, mit der in 18.5 die Rechnung beginnt, eben nicht in der Aufgabenstellung genannt war. Eine 9 für den Durchmesser wäre dann angegeben gewesen, wenn die nb.t ein Halbkreis wäre. Da aller Wahrscheinlichkeit nach die 9 aber bereits durch Verdoppelung aus einer $4 \frac{1}{2}$ entstanden ist, kann die $n b . t$ nur ein Halbzylinder sein. Da überdies bei zwei gleichen Zahlen das Ausfallen einer der beiden wahrscheinlicher ist als das Ausfallen einer von zwei unterschiedlichen Zahlen, halte ich es für sicher, daß in der Aufgabenstellung der Vorlage des Moskauer Papyrus tatsächlich die für die Berechnung der gekrümmten Halbzylinderaußenfläche benötigten Angaben von jeweils $4 \frac{1}{2}$ für th- $r$ ? und ${ }^{c} d$ gestanden haben.

Mit all diesen Überlegungen paßt auch das leider arg beschädigte Wort in 18.6 zusammen. $\mathrm{Daß}$ eine Lesung inr kaum zulässig ist, hat schon Peet gezeigt ${ }^{32}$. Dagegen trägt seine Ergänzung ip.t den Spuren Rechnung: If $^{33}$. So wie wir die $n b . t$ verstehen, als Halbzylinder, wird die Aussage der Zeilen 18.5f. leicht einsichtig: Ein Halbzylinder ist ja gerade die Hälfte eines tönnchenförmigen Scheffelmaßes. Tatsächlich ist das Wort ip.t schon in der 11. Dynastie belegr ${ }^{34}$, in den sog. Heḳanachte Papers nämlich ${ }^{35}$. Das Wort kommt z. B. VI.12 und VII.4 vor. Gerade der zweite Beleg, der mit den Vermerken ,in der Stadt“ bei zwei der folgenden Einträge parallel geht, belegt, daß ip.t hier im weitesten Sinne einen Aufenthaltsort (für Emmer) angibt. Es kann hier also nicht das abstrakte Maß „Scheffel“, sondern nur das Meßgefäß meinen. Und dieses Meßgefäß ist eben etwa zylindrisch. Man beachte ferner, daß auch koptisch o $\varepsilon \imath \pi \varepsilon$ nicht nur das Maß, sondern auch das Gefäß, in dem gemessen wird, bezeichnet ${ }^{36}$.

Selbst wenn das fragliche Wort anders wiederherzustellen sein sollte, dürfte eines sicher sein: Ein Wort für Kreis oder Kugel ist es nicht. Die nb.t, die hier berechnet wird, ist vielmehr ein halber Zylinder, dessen gekrümmte Mantelfläche Gegenstand der Aufgabe 10 des Moskauer mathematischen Papyrus ist. Daß den Ägypter gerade die halbe Zylinderfläche interessierte, muß nicht so sehr verwundern, wenn man daran denkt, daß mit nb.t auch ein Bergrücken oder vielleicht auch ein Gewölbe bezeichnet wird. Im ersten Fall schaut ja gewissermaßen nur die eine Hälfte eines Zylinders aus der Erde heraus, im zweiten haben wir es naturgemäß ebenfalls nur mit einem halben Zylinder zu tun, den man von innen her betrachtet. Meint nb.t einen Korb, liegt der Fall analog.

Neugebauers Einwand ${ }^{37}$, daß die nb.t kein Korb sein könne, da die Maßangaben sicher in Ellen gemeint sind, die nb.t also viel zu groß für einen Korb wäre, übersieht, daß einerseits die ursprüngliche Bedeutung von nb.t nicht „Korb“ sein muß, sondern „Bergrücken“ sein

\footnotetext{
${ }^{32}$ Peet op. cit. S. $102 \mathrm{f}$.

${ }^{33}$ Peet op. cit. S. 105; vgl. die ältere Photographie bei Struve op. cit. S. 164.

${ }^{34}$ Wb I S. 67,6ff. kennt nur Belege seit der 18. Dynastie. Ein weiterer früher Beleg ist die Prophezeiung des Neferti XIc (ed. Helck, W.: Die Prophezeiung des Nfr.tj. Wiesbaden 1970 (= Kleine ägyptische Texte), S. 42).

${ }^{35}$ Ed. James, T. G. H.: The Heḳanakhte Papers and Other Early Middle Kingdom Documents. New York 1962 (= Publications of the Metropolitan Museum of Art - Egyptian Expedition 19).

${ }^{36}$ Crum, W. E.: A Coptic Dictionary. Oxford 1979, S. 256 a.

${ }^{37}$ Neugebauer, O.: Vorlesungen ... S. 136.
} 
$\operatorname{kann}^{38}$. Andererseits wird das Wort hier ohne Frage als mathematischer Terminus verwendet und muß mit einem Korb im eigentlichen Sinne längst nichts mehr zu tun haben.

Wenn die halbkreisförmigen Seitenflächen nicht mitberechnet werden, so dürfte das durchaus dem ägyptischen Verständnis von einer nb.t entsprechen. An den Dekorationen im Grab Ramses' VI. beispielsweise, auf das ich schon hingewiesen habe, sieht man in aller Deutlichkeit, daß diese fast halbkreisförmigen Flächen nicht zur gewölbten Decke gehören ${ }^{39}$. Ich sehe somit keinen Grund, an der schon von Peet vorgetragenen Deutung der nb.t im Moskauer mathematischen Papyrus als Halbzylinder zu zweifeln.

ANHANG: TRANSKRIPTION UND ÜBERSETZUNG

$$
1 \infty_{0}^{\infty} 1 \text { ma }
$$

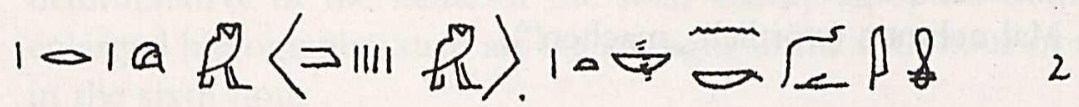

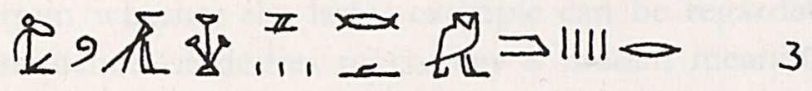

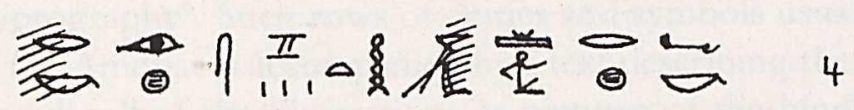

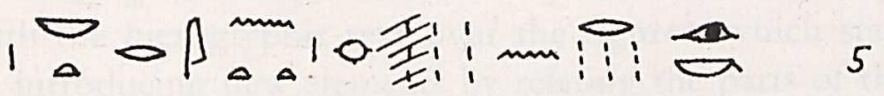

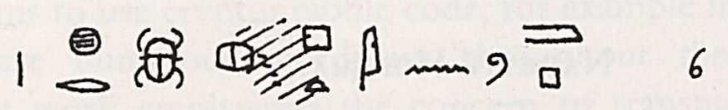

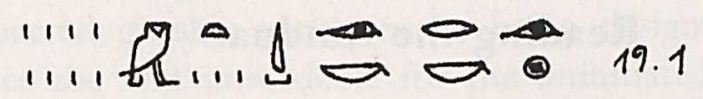

$$
\begin{aligned}
& \because 11 \operatorname{man}: 000
\end{aligned}
$$

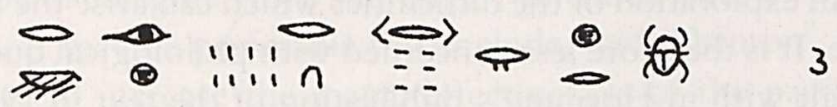

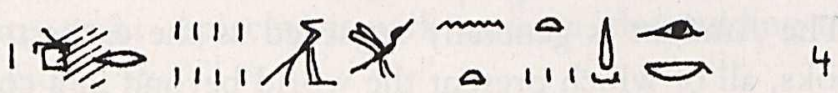

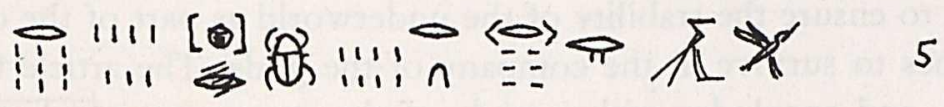

$$
\begin{aligned}
& \text { चIII) }
\end{aligned}
$$

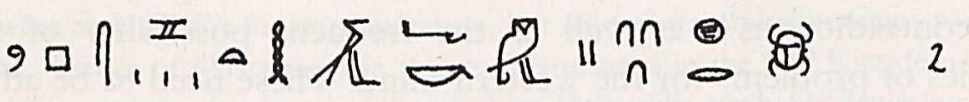

$$
\begin{aligned}
& \text { 热 视 } 3
\end{aligned}
$$

\footnotetext{
${ }^{38}$ Oder noch allgemeiner ,halbzylindrische Auswölbung“.

${ }^{39} \mathrm{Vgl}$. Hornung loc. cit.
} 


\section{1. „Form der Berechnung einer nb.t:}

2. Wenn man dir nennt eine nb.t 〈von 41/2〉 (Maßeinheiten) als Mündung

3. auf $4 \frac{1}{2}$ (Maßeinheiten) als Längskante - bitte

4. laß mich ihre Fläche wissen! Du sollst

5. dann $1 / 9$ von 9 ermitteln - denn was eine $n b . t$ anbelangt,

6. so ist sie die Hälfte eines Scheffels - ergibt also 1.

19.1. Du sollst dann den Rest als 8 ermitteln.

2. Du sollst dann $1 / 9$ von 8 ermitteln,

3. ergibt also $2 / 3+\left\langle{ }^{1}\right\rangle_{6}+1 / 18$. Du sollst

4. dann den Rest von dieser 8 nach

5. diesem $2 / 3+\left\langle{ }^{1}\right\rangle_{6}+1 / 18$ ermitteln; ergibt [al]so $71 /$.

20.1. Du sollst dann $7 \frac{1}{9} 4 \frac{1}{2} \mathrm{Mal}$ nehmen (wörtlich ,machen“);

2. ergibt also 32. Siche, das ist ihre Fläche.

3. Du hast richtig gefunden“". 